\title{
RAZUMIKHIN'S METHOD IN THE QUALITATIVE THEORY OF PROCESSES WITH DELAY ${ }^{1}$
}

\author{
ANATOLY D. MYSHKIS \\ Moscow State University of Communications \\ Department of Mathematics \\ Obraztsova St. 15, Moscow 101475, Russia
}

(Received January, 1995; revised March, 1995)

\begin{abstract}
B.S. Razumikhin's concept in the qualitative theory of systems delay is clarified and discussed. Various ways of improvements of stability conditions are considered. The author shows that the guiding role of Lyapunov functions and demonstrates Razumikhin's method as a practical case of continuous version of the mathematical induction. Several examples demonstrate the obtained results.
\end{abstract}

Key words: Functional-Differential Equation of Retarded Type, Qualitative Theory, Systems with Delay, Razumikhin's Method, Stability Solution, Lyapunov Function, First Breakdown, Guiding Function.

AMS (MOS) subject classifications:34C99, 34D20, 58F10, 93D05, 93D20.

\section{INTRODUCTION}

B.S. Razumikhin proposed in 1956 [41] a method to investigate stability of solutions of systems with delays. This method is based on application of Lyapunov functions in combination with the general concept of "impossibility of the first breakdown" (see Section 7). This method was developed later both by Razumikhin himself (in the most explained form in monograph [38]) and by other authors (see, in particular, J. Hale's books [13,14]). Sometimes this method is regarded as more superior to N.N. Krasovskii's method of functionals that seems to be unjustified (see Section 9).

Razumikhin's method essentially extends stability theorems in Lyapunov's sense. In this case, it is necessary to pass from "guiding Lyapunov's functions" to arbitrary guiding functions.

These and related questions will be considered in the paper. Since only basic concepts will be discussed, the statements will be presented not in a general form and without detailed proofs.

\section{GUIDING FUNCTIONS FOR SYSTEMS WITHOUT DELAY}

Let the state of some autonomous system be defined by the point $x=\left(x_{1}, \cdots, x_{n}\right)$ of $n$ dimensional domain $D$, whereas its evolution is governed by the differential law, i.e. by the system of ordinary differential equations (ODEs)

\footnotetext{
${ }^{1}$ With partial support from International Science Foundation (grant MH 9000), Russian Foundation of Fundamental Researches (grant 93-0100884), and American Mathematical Society. 


$$
\dot{x}=f(x)
$$

with smooth (i.e. continuously differentiable) right-hand side. The solutions $t \mapsto x(t)$ of this system are interpreted naturally as trajectories in the phase space $D$.

Let $V: D \rightarrow \mathbb{R}$ be a given smooth function, i.e. $V(x)$ is a scalar characteristic of a physical system considered at state $x$. Then the change rate of this characteristic at state $x$ is a derivative of the composite function and is equal to:

$$
d V(x(t)) / d t=\sum_{i=1}^{n} V_{x_{i}}(x) \dot{x}_{i}=(\operatorname{grad} V(x), \dot{x})=(\operatorname{grad} V(x), f(x)),
$$

where $\operatorname{grad} V(x):=\left(V_{x_{1}}(x), \cdots, V_{x_{n}}(x)\right)$, and $(\cdot, \cdot)$ means a scalar product of vectors. This derivative is called the derivative of the function $V$ taken by virtue of system (1); we shall denote it by

$$
\dot{V}^{(1)}(x):=(\operatorname{grad} V(x), f(x))
$$

(Index (1) in the left-hand side indicates the system of equations by virtue of which the derivative is taken.)

Thus, if the value $x(t)$ of the solution is known for some $t$, then it is possible to get the value $d V(x(t)) / d t$ for this $t$ directly, without solving system (1). This simple property is crucial for application of guiding functions and, in particular, guiding Lyapunov functions.

We shall call a smooth function $V: D \rightarrow \mathbb{R}$ a guiding function for the system (1) if $\dot{V}^{(1)}(x) \leq 0$ in $D$; in other words, if it is a decreasing function in the weak sense (i.e. it can have intervals of constancy) along any trajectory of this system. The guiding function for a physical system is like a wind which drives it from the states with higher levels (of values of $V(x)$ ) to the states with lower levels; in any case, this level can remain constant, but cannot increase. It is natural that the knowledge of the guiding function for some system allows us to make essential conclusions about the character of its evolution.

It was A.M. Lyapunov who for the first time began to apply systematically guiding functions in his famous studies [31] of stability theory and it is usual to call such functions Lyapunov functions. We are going to use this term in a wider sense: namely, we call a Lyapunov function in $\mathbb{R}^{n}$ any scalar smooth function $V$, defined in some neighborhood $G$ of the origin $0 \in \mathbb{R}^{n}$, such that $V(0)=0, V(x)>0(\forall x \in G \backslash\{0\})$.

Let us recall Lyapunov's theorems on stability and asymptotic stability for system (1). (Lyapunov proved them for the general case of non-autonomous systems of ODEs.)

Theorem 1. Let $0 \in G, f(0)=0$ and assume a Lyapunov function $V: G \rightarrow \mathbb{R}$ exists, for which $\dot{V}^{(1)}(x) \leq 0(\forall x \in G)$ (i.e. which is guiding for (1)). Then the solution $x(t) \equiv 0$ of system (1) is stable in the sense of Lyapunov.

Theorem 2. Assume, in addition to the conditions of Theorem 1, that $\dot{V}^{(1)}(x)<0$ $(\forall x \in G \backslash\{0\})$. Then the solution $x(t) \equiv 0$ of system (1) is asymptotically stable in the sense of Lyapunov.

These statements have a simple interpretation. The level surfaces of Lyapunov function $V$ (i.e. surfaces in $\mathbb{R}^{n}$ defined by the equation $V(x)=$ const.) are similar to the family of concentric spheres with center 0 and any sufficiently small radii. It follows from the negativeness of $\dot{V}^{(1)}$ that all those surfaces intersect trajectories of system (1) towards the interior. (If $\dot{V}^{(1)} \leq 0$ then the trajectories can slide along the level surfaces). Hence, the validity of the theorems mentioned becomes intuitively clear.

M.A. Krasnosel'skii was the first in 1958 to apply guiding functions for studying different 
problems of the qualitative theory of ODEs. Let us give one example of such an application for system (1).

Theorem 3. Let a function $f$ be defined on $\mathbb{R}^{n}$ and let the smooth function $V: G \rightarrow \mathbb{R}$ exist in some neighborhood $G$ of infinity (i.e. $G$ contains all points $x$ with sufficiently large $|x|$ ) such that $V(x) \rightarrow \infty$ as $x \rightarrow \infty$ and $\dot{V}^{(1)}(x)<0(\forall x \in G)$. Then system (1) is dissipative, i.e. there exists an $r>0$ such that any solution of system (1) belongs to the sphere $|x|<r$, starting at some instant dependent on initial conditions.

The intuitive interpretation of this theorem is similar to that of Theorems 1 and 2 mentioned above, but level surfaces are similar now to the family of spheres of any sufficiently large radius.

It should be noted that in some papers, guiding functions with values in some ordered vector space are also considered; e.g. Lyapunov vector functions successfully applied by V.M. Matrosov. Throughout this paper, we shall consider only scalar guiding functions.

\section{FUNCTIONAL DIFFERENTIAL EQUATIONS OF RETARDED TYPE}

These equations (briefly RDEs) and their systems describe processes whose evolution rate is defined at any instant of time by their state not only at that instant but also at preceding instants. In other words, we consider processes with lags (concentrated delay) or with aftereffects (distributed delay). RDEs are widespread now in different mathematical applications such as control problems, population dynamics, and in other branches of science such as biology, medicine, economics, viscoelasticity, etc.

Let us investigate an autonomous system of the 1st order RDEs with single discrete constant delay:

$$
\dot{x}(t)=f(x(t), x(t-h)), h=\text { const. }>0
$$

Here, $x(t)$ takes values in $\mathbb{R}^{n}$, whereas the function $(x, y) \mapsto f(x, y)$ with $2 n$ scalar arguments is defined for all $x, y \in \mathbb{R}^{n}$ and is smooth. However, all considerations can be extended mutatis mutandis to other forms of RDEs.

Let us assume that we apply $\operatorname{RDE}(2)$ beginning with some initial instant $t=t_{0}$. Since we have $t-h \in\left[t_{0}-h, t_{0}\right]$ for $t \in\left[t_{0}, t_{0}+h\right]$, and since $x(t-h)$ is present in system (2), the values of the unknown solution $x(\cdot)$ must be given on the interval $\left[t_{0}-h, t_{0}\right]$ as an initial condition, a kind of prehistory.

It can be proved that, for any continuous initial function $t \mapsto x(t)\left(t_{0}-h \leq t \leq t_{0}\right)$, the corresponding solution of system (2) exists and is unique on some interval $t_{0} \leq t<T(\leq \infty)$ where $T$ depends on the initial function. This solution depends continuously (in a sense of uniform deviation) on the initial function on any interval $\left[t_{0}, T_{1}\right] \subset\left[t_{0}, T\right)$. A smoothing of the solution takes place as $t$ increases: it is smooth for $t_{0} \leq t<T$; if $T>t_{0}+h$, then it is twice as smooth for $t_{0}+h \leq t<T$; it $T>t_{0}+2 h$ and $f$ is twice as smooth, then $x(\cdot)$ is thrice as smooth for $t_{0}+2 h \leq t<T$ and so on. Besides, if $T<\infty$, then $|x(t)| \rightarrow \infty$ for $t \rightarrow T^{-}$.

An important peculiarity of RDEs should be noted: if the initial condition is given, the solution exists generally speaking in the direction of increasing $t$ only; the problem of solution construction is found to be incorrect in the direction of decreasing $t$.

According to what has been said, it is natural to take the space of all continuous functions on some closed interval of length $h$ valued in $\mathbb{R}^{n}$ and a norm of the uniform deviation as phase space corresponding to system (1); N.N. Krasovskii was the first to propose this in 1956 [28] and it is generally accepted now. One usually takes $[-h, 0]$ as such an interval. Then, the corresponding 
space is denoted by $(C[-h, 0])^{n}$; it is sometimes called Krasovskii's space associated with the system of RDEs (2).

The trajectory in the space $K:=(C[-h, 0])^{n}$ corresponds to each solution $t \mapsto x(t)$ $\left(t_{0}-h \leq t<T\right)$ of system (2) by the following rule: each $t \in[0, T)$ corresponds to the function $\theta \mapsto x_{t}(\theta):=x(t+\theta)(-h \leq \theta \leq 0)$ as an element (point) of $K$. Exactly one trajectory starts at each point $\phi \in K$. It corresponds to the solution of system (2) with initial function

$$
x(t)=\phi\left(t-t_{0}\right) \quad\left(t_{0}-h \leq t \leq t_{0}\right)
$$

and the same trajectory corresponds to different values of $t_{0}$ but passing with translation in time. Thus the phase space $K$ and the trajectories in it for system (2) are similar in many aspects to the phase space $\mathbb{R}^{n}$ and the trajectories for ODE (1); but an important distinction still exists: the space $K$ is infinite-dimensional.

The concepts of stability and asymptotical stability of the solution of problem (2),(3) in the sense of Lyapunov, are introduced quite similar to the system of ODEs (1). In what follows, we shall omit the words "in the sense of Lyapunov."

\section{GUIDING FUNCTIONALS FOR SYSTEMS OF RDES}

Let the scalar functional $P: K \rightarrow \mathbb{R}$ be given in the space $K=(C[-h, 0])^{n}$, i.e. the number $P(\phi) \in \mathbb{R}$ corresponds to any $\phi \in K$. We shall consider only continuous functionals, i.e., it is assumed that the uniform convergence of the sequence $\phi^{1}, \phi^{2}, \ldots$ implies the convergence of the numerical sequence $P\left(\phi^{1}\right), P\left(\phi^{2}\right), \cdots$. Then it is easy to verify that if the function $x:\left[t_{0}-h\right.$, $T] \rightarrow \mathbb{R}^{n}\left(t_{0}<T\right)$ is continuous, then the function $t \mapsto P\left(x_{t}\right)\left(t_{0} \leq t<T\right)$ is also continuous.

The derivative of the functional $P$ taken by virtue of system (2) is defined analogously to that of system of ODEs (1) in Section 2. Namely, if $\phi \in K$ and $x^{\phi}$ is a solution of system (2) under the initial condition (3), then

$$
\dot{P}^{(2)}(\phi):=\varlimsup_{\Delta t \rightarrow 0}+\frac{1}{\Delta t}\left[P\left(x_{t_{0}}^{\phi}+\Delta t\right)-P(\phi)\right] .
$$

Here $\varlimsup_{\Delta t \rightarrow 0}+$ means the largest of the limits as $0<\Delta t \rightarrow 0$ (it may be a nonunique limit of this value). $\dot{P}^{(2)}$ is a generalized functional $K \rightarrow \mathbb{R} \cup\{-\infty, \infty\}$ in the sense that it can assume $\pm \infty$ along with finite values.

From formula (4) and the autonomy of system (2) it follows that the solution $x(\cdot)$ of problem (2), (3) satisfies the equation

$$
\varlimsup_{\Delta t \rightarrow 0}+\frac{1}{\Delta t}\left[P\left(x_{t+\Delta t}\right)-P\left(x_{t}\right)\right]=\dot{P}^{(2)}\left(x_{t}\right) \quad\left(t_{0} \leq t<T\right)
$$

The limit on the left-hand side is called upper right derivative of the function $t \mapsto P\left(x_{t}\right)$. The simple known lemma below makes use of this derivative.

Lemma 1. If the function $g:[\alpha, \beta] \rightarrow \mathbb{R}(\alpha<\beta)$ is continuous and its upper right derivative $\dot{g}^{u r}(t) \leq 0$ (resp. $\geq 0$ ) for $\alpha \leq t<\beta$, then the function $g$ is nonincreasing (resp. nondecreasing) on $[\alpha, \beta]$. If in addition the set $\left\{t: \dot{g}^{u r}(t)=0\right\}$ does not contain intervals, then the function $g$ is decreasing (resp. increasing) on $[\alpha, \beta]$.

Analogously to ODEs, the functional $P$ is called a guiding functional in some domain $H \subseteq K$ for some system of RDEs $(2)$ if $\dot{P}^{(2)}(\phi) \leq 0(\forall \phi \in H)$. By virtue of Lemma $1, P$ is then a 
nonincreasing function of $t$ along any arc of the trajectory of system (2) entirely contained in $H$.

Guiding "Lyapunov functionals" are an important example of guiding functionals; such functionals were introduced by N.N Krasovskii ([28], see also [27]) and were successfully used by him and other authors in the theory of RDEs. We shall cite Krasovskii's theorems, which are analogous to Lyapunov's theorems pointed out in Section 2 and are adapted to system (2).

Theorem 4. Let $f(0,0)=0$ and assume there exists a functional $P$ in some neighborhood $H$ of the zero function $\phi=0$ for which $P(0)=0, P(\phi)>0(\phi \neq 0)$, and for each $\epsilon>0$ there is a $\delta>0$ such that $|P(\phi)|<\delta$ implies that $|\phi(0)|<\epsilon$ and $\dot{P}^{(2)}(\phi) \leq 0(\forall \phi \in H)$. Then the solution $x(t) \equiv 0$ of system (2) is stable.

Theorem 5. Assume that, in addition to the conditions of Theorem 4, $\sup \left\{\dot{P}^{(2)}(\phi)\right.$ : $P(\phi)>\delta\}<0$ for any sufficiently small $\delta>0$. Then the solution $x(t) \equiv 0$ of system $(2)$ is asymptotically stable.

The efficiency of Lyapunov's functionals in RDEs stability, for general and particular cases, was confirmed in $[13,14,25,26]$.

The following theorem can be used, for example, as an analog of Theorem 3.

Theorem 6. Let the functional $\phi \rightarrow P(\phi)$ be defined for all $\phi \in K$ with sufficiently large $|\phi(0)|$, $P(\phi) \rightarrow \infty$ as $|\phi(0)| \rightarrow \infty$, and $\sup \left\{\dot{P}^{(2)}(\phi): P(\phi)>M\right\}<0$ for any sufficiently large $M$. Then there exists $r>0$ such that the solution of problem (2),(3) will be contained in the sphere $|x|<r$ for any initial function, beginning at some $t$.

\section{DIRECT APPLICATION OF LYAPUNOV FUNCTIONS TO RDES}

Prior to N.N. Krasovskii's papers on Lyapunov's functionals, L.E. El'sgol'ts [6] considered the stability problem of the solution $x(t) \equiv 0$ of RDEs exactly as that for ODEs by proving that the function $t \mapsto V(x(t))$ is decreasing. Here $V$ is some Lyapunov function and $x(\cdot)$ is any perturbed solution. He showed that it is only possible in some comparatively rare special cases.

We shall show this on the example of system (2) with $f(0,0)=0$ for $n=1$, i.e. when it turns into a scalar equation. Let us assume that $V(x)=x^{2}$ which is a typical Lyapunov function for $n=1$. Then we have for any solution $x(\cdot)$ of equation $(2)$ that

$$
[V(x(t))]^{\cdot}=2 x(t) \dot{x}(t)=2 x(t) f(x(t), x(t-h)) .
$$

For the validity of inequality $[V(x(t))] \leq 0$, we need to require that $x f(x, y)$ be nonpositive for all sufficiently small $|x|$ and $|y|$. It follows then that $f(0, y) \equiv 0$ which essentially restricts the class of equations considered.

It should be noted that the function $t \mapsto V(x(t))$, with a given $V$, can be written in the form $t \mapsto P\left(x_{t}\right)$, if we define the functional $P$ by $P(\phi):=V(\phi(0))$, because $P\left(x_{t}\right)=V\left(x_{t}(0)\right)=V(x(t))$. We get for this functional $\dot{P}^{(2)}(\phi)=(\operatorname{grad} V(\phi(0)), f(\phi(0), \phi(-h)))$.

\section{THE MAIN IDEA OF B.S. RAZUMIKHIN}

The method proposed by B.S. Razumikhin [41] allowed to "rehabilitate" an application of Lyapunov functions to RDEs to a considerable extent. This application was found in some cases simpler and more visual than an application of general functionals. 
The example from Section 5 helps understand easily the idea of Razumikhin's method. We need the inequality $[V(x(t))]^{\cdot} \leq 0$ in order to prove that a solution starting in a small neighborhood of the zero function remains in it in the future. However, it does not require for the latter that the value of $V(x(t))$ be decreasing (or at least nonincreasing) all the time; it is sufficient that the trajectory, which corresponds to the solution, will not cross the boundary of this neighborhood. Hence, if a neighborhood has the form $V(x)<\delta(\delta=$ const. $>0)$, it would be sufficient to require that $[V(x(t))]^{\circ}<0$ when $V(x(t))=\delta$ and $V(x(t-h))<\delta$, because the last inequality holds when the trajectory reaches the boundary for the first time. We show later that the inequality $\dot{V}<0$ can be replaced here by $\dot{V} \leq 0$. Thus, for stability of the zero solution, the inequality $x f(x, y) \leq 0$ need not be valid for all $x$ and $y$ with sufficiently small modulo, but only for those with $|y|<|x|$. This (weaker) restriction extends considerably the number of cases for which stability of the zero solution of equation (2) can be proved by Lyapunov functions.

Let us consider, as a particular case, the scalar RDE

$$
x(t)=a x(t)+b x(t-h) \quad(a, b=\text { const. }) .
$$

Stability of the solution $x(t) \equiv 0$ is guaranteed by approach in Section 5 , with $V(x)=x^{2}$, only in the trivial case $b=0$ and $a \leq 0$. Using Razumikhin's method, we conclude that the stability is ensured if $x(a x+b x) \leq 0$ for $|y|<|x|$, i.e. always when $|b| \leq-a$. (Of course, as for any linear system of RDEs, from stability of some solution of equation (5) it follows the stability of any other solution, i.e. here it is natural to consider the stability of this system; the asymptotic stability of linear systems is analogously considered.)

\section{7. "IMPOSSIBILITY OF THE FIRST BREAKDOWN"}

The idea of Razumikhin's method can also be explained in more general situations. Let us consider a continuous evolution of some system, starting from some instant, and we wish to prove that it possesses some property $S$ all the time. Let this property be stable, i.e. if the system possesses it in some state, then it would do so for all sufficiently close states.

Two ways can be used to prove, by contradiction, the presence of property $S$. First, it can be assumed simply that the property $S$ is absent at some instant, and try to arrive at a contra-. diction. Second, it can be proved that $S$ takes place at the initial instant, and then deduce a contradiction considering the first instant of breakdown of this property. It is clear that if we are able to prove that the first breakdown is impossible, then it would always be impossible.

The second approach is in some sense analogous to the mathematical induction method for continuous processes. It is effective for the cases when the assumption about the validity of the property $S$ at all instants preceding the considered one, gives an information which facilitates to get a contradiction. Of course, this approach was used by many authors in different branches of mathematics. It is crucial for Razumikhin's method along with the idea of a guiding function.

\section{PRECISE FORMULATION}

To apply Razumikhin's method, one can use the following lemma, which is a refinement of a particular case of J. Kato's theorem [21], but can easily be proved independently.

Lemma 2. Let the function $g:[\alpha-h, \beta] \rightarrow \mathbb{R}$ be continuous for some $h>0, \alpha<\beta \leq \infty$, and $\dot{g}^{u r}(t) \leq 0$ for all values $t \in[\alpha, \beta)$ for which $g(s)<g(t) \quad(\forall s \in[\alpha-h, t))$. Then $g(t)$ $\leq \max _{s \in[\alpha-h, \alpha)} g(s)(\alpha \leq t<\beta)$. 
Applied to system (2) with $f(0,0)=0$, this lemma leads to the theorem:

Theorem 7. Assume the Lyapunov function $V: G \rightarrow \mathbb{R}$ exists with $x, y \in G, V(y)<V(x)$ imply

$$
(\operatorname{grad} V(x), f(x, y)) \leq 0 .
$$

Then the solution $x(t) \equiv 0$ of system (2) is stable.

For a proof, one must set $g(t)=V(x(t))$, where $x(\cdot)$ is any solution of system (2) with initial function $\phi$ sufficiently close to zero, and apply Lemma 2 . We see that even a stronger statement for such solutions holds: $V(x(t)) \leq \max _{\left[t_{0}-h, t_{0}\right]} V(\phi)$. However, in general, the monotonicity of the function $t \mapsto V(x(t))$ does not hold true, unlike the situation arising under conditions of Theorem 1. Thus the function $V$ turns out to be not a guiding function in the restricted sense, but a " barrier" function.

It should be noted that the function $t \mapsto g(t)$, defined in the proof of Theorem 7 , is smooth for $t \geq \alpha:=t_{0}$. Lemma 2 follows easily under this additional assumption from the following particular case of A. Sard's theorem: a set of stationary values of a smooth function of a single argument has the everywhere dense complement.

Theorem 7 is directly extended with natural modifications to any autonomous systems of 1st order RDEs, both with discrete and continuous delays, and to the non-autonomous case; see e.g. Section 5.4 of [13] and the references therein. Besides, if a non-autonomy of the system appears only in the dependence of the delay on $t$ or even on the unknown solution, then no changes in the formulation and the proof of the theorem are required.

\section{CONNECTION BETWEEN RAZUMIKHIN'S METHOD AND LYAPUNOV FUNCTIONALS}

Let a smooth function $V: \mathbb{R}^{n} \rightarrow \mathbb{R}$ be given. Define the functional $P: K \rightarrow \mathbb{R}$ as follows

$$
P(\phi)=\max _{-h \leq \theta \leq 0} V(\phi(\theta)) \quad(\forall \phi \in K) .
$$

It is not difficult to verify that

$\dot{P}^{(2)}(\phi)= \begin{cases}\leq 0, & \text { if } V(\phi(0))<P(\phi), \\ =\max \{(\operatorname{grad} V(\phi(0)), f(\phi(0), \phi(-h))), 0\}, & \text { if } V(\phi(0))=P(\phi) .\end{cases}$

Thus, $\dot{P}^{(2)}(\phi)>0$ if only if

$$
V(\phi(0))=\max _{-h \leq \theta \leq 0} V(\phi(\theta)) \quad \text { and } \quad(\operatorname{grad} V(\phi(0)), f(\phi(0), \phi(-h)))>0 .
$$

The function $V$ can be defined on some domain $G \subset \mathbb{R}^{n}$. The functional $P$ is then defined for $\phi \in K$ with values in $G$. Its properties, pointed out here, remain valid.

If the function $V$ is such as in Theorem 7, then relations (7) are inconsistent. Indeed, if they are valid for some function $\phi \in K$, then we could obtain the inequality $V(\phi(-h))<V(\phi(0))$ making $\phi$ arbitrarily small. At the same time, the second relation in (7) remains valid. It contradicts the condition of Theorem 7 . Hence $\dot{P}^{(2)}(\phi) \leq 0$ for all $\phi$. So, if $f(0,0)=0$, then all the conditions of Theorem 4 are satisfied and therefore, the solution $x(t) \equiv 0$ of equation (2) is stable.

Thus, Razumikhin's method can be considered as a particular case of the method of 
Lyapunov functionals. But this particular case possesses a feature which makes its independent study quite natural. This feature is based, first of all, on the possibility to apply the guiding functions in $\mathbb{R}^{n}$ (with $n=1,2$ etc. in many examples) which is well elaborated for ODEs.

\section{ASYMPTOTIC STABILITY}

Asymptotic stability of system (2) zero solution can be proved under a stronger assumption than that of Theorem 7. Consider an example of such statement.

Theorem 8. Assume, in addition to the conditions of Theorem 7 , that for any sufficiently small $p>0$, there exists $q>p$ such that, if $V(x)=p, V(y)<q$, then $(\operatorname{grad} V(x), f(x, y))<0$. Then the solution $x(t) \equiv 0$ of system (2) is asymptotically stable.

Indeed, if the solution $t \mapsto x(t)$ of system (2) is modulo sufficiently small and does not tend to zero as $t \rightarrow \infty$, then we denote $p:=\varlimsup_{t \rightarrow \infty} V(x(t))$, and apply the additional condition of Theorem 7 which leads to the contradiction.

In particular, we obtain for $n=1$, taking $V(x)=x^{2}$, that the condition $|b|<-a$ is sufficient for the asymptotic stability of equation (5).

Remark. If the function $V$ is defined on the whole $\mathbb{R}^{n}$ in Theorem 8 , and $V(x) \rightarrow \infty$ as $|x| \rightarrow \infty$, and $p>0$ can be arbitrary, then the solution of system (2) tends to zero as $t \rightarrow \infty$ for arbitrary initial function.

\section{REFINEMENT OF ESTIMATIONS}

Razumikhin also notes that many parameters estimates of RDEs, which guarantee stability, can be made much more accurate by constructing a set of points where the validity of inequalities (6) is required. Let us formulate a relevant theorem for system (2) with $f(0,0)=0$.

Theorem 9. Assume that the Lyapunov function $V: G \rightarrow \mathbb{R}$ and a number $N>0$ exist such that the inequality

$$
(\operatorname{grad} V(x(0)), f(x(0), x(-h))) \leq 0,
$$

holds true for any continuous function $x(\cdot):[-(N+1) h, 0] \rightarrow G$ for which $x(t) \in G, V(x(t))$ $<V(x(0))(-(N+1) h \leq t<0)$, and which satisfies system (2) for $-N h \leq t \leq 0$. Then the solution $x(t) \equiv 0$ of system $(2)$ is stable.

Indeed, let $\epsilon>0$ be given with $\{x:|x|<\epsilon\} \subset G$ and $\phi \in K, V(\phi(t))<\delta(-h \leq t \leq 0)$, where $\delta>0$ is sufficiently small. Then the inequality $V(x(t))<\epsilon\left(t_{0} \leq t \leq t_{0}+N h\right)$ for the solution $x(\cdot)$ of problem (2), (3) follows from continuous dependence of the solution on the initial function. The impossibility of the breakdown of this inequality for $t>t_{0}+N h$ can be justified as in Theorem 7.

\section{EXAMPLE}

Let us show how Theorem 9 can be applied to the scalar equation

$$
\dot{x}(t)=-b x(t-h) \quad(b=\text { const. }>0),
$$

with $V(x)=x^{2}$. We are following Razumikhin's presentation with some refinements. 
The application of Theorem 7 gives nothing in this example that can be seen from the consideration of equation (5) in Section 6. Let us apply Theorem 9. Observe that inequality (8) takes the form

$$
[2 x(0)][-b x(-h)] \leq 0, \text { i.e. } x(0) x(-h) \geq 0 .
$$

Let us take at first $N=1$; then inequality (10) must be proved for any continuous function $x(\cdot):[-2 h, 0] \rightarrow \mathbb{R}$, for which $|x(t)|<|x(0)|(-2 h \leq t<0)$, and equation (9) is satisfied for $-h \leq t \leq 0$.

If $x(0)=0$ then the validity of $(10)$ is obvious. Therefore, let $x(0)>0$ (if $x(0)<0$, we can multiply $x(\cdot)$ by -1 , which does not affect $(10))$.

It follows by virtue of equation (10) from inequality $|x(t)|<x(0)(-2 h \leq t \leq-h)$ that

$$
\dot{x}(t)<b x(0) \quad(-h<t<0) .
$$

Now integrating it from $-h$ to 0 , we obtain $x(0)-x(-h)<b x(0) h$. Hence, $x(-h)$ $>(1-b h) x(0)$.

We see that if $b h \leq 1$, then inequality (10) holds true, and therefore, the condition $b h \leq 1$ is sufficient for the stability of equation (9).

This restriction can be essentially weakened, if we put $N=2$. Inequality (10) must be verified now for any continuous function $x(\cdot):[-3 h, 0] \rightarrow \mathbb{R}$, for which $|x(t)|<|x(0)|$ $(-3 h \leq t<0)$, and equation (9) is satisfied for $-2 h \leq t \leq 0$.

We can assume again $x(0)>0$. Let us assume that $(10)$ is not fulfilled, i.e. $x(-h)<0$; then $b h>1$ by virtue of considerations carried out for $N=1$. We obtain by integrating (9) from any $t \in[-2 h,-h]$ to $-h$ that

$$
|x(-h)-x(t)| \leq b x(0)(-h-t) \quad(-2 h \leq t \leq-h) .
$$

It follows from here that

$$
x(t) \geq \max \{-x(0), x(-h)-b x(0)(-h-t)\} \quad(-2 h \leq t \leq-h) .
$$

Let $t=g$ be the value for which both quantities under max equal each other, i.e.

$$
g:=-h-\frac{1}{b}-\frac{x(-h)}{b x(0)}
$$

Since $b h>1$, then $-2 h<g<-h$ and therefore inequality (11) can be rewritten as follows:

$$
x(t) \geq\left\{\begin{array}{lc}
-x(0), & (-2 h \leq t \leq g), \\
x(-h)+b x(0)(h+t), & (g \leq t \leq-h) .
\end{array}\right.
$$

Using this estimation we obtain by integrating both sides of equation (9) from $-h$ to 0 that

$$
\begin{aligned}
x(0)-x(-h) & =-b\left[\int_{-2 h}^{g} x(t) d t+\int_{g}^{-h} x(t) d t\right] \\
& \leq-b\left\{-x(0)(g+2 h)+x(-h)(-h-g)+b x(0)\left[-\frac{1}{2}(h+g)^{2}\right]\right\} .
\end{aligned}
$$

Substituting it into the right-hand side of (12) for $g$ and grouping similar terms, we get:

from which

$$
x(0)-x(-h) \leq-b h x(0)-\frac{1}{2} x(0)-x(-h)-\frac{1}{2 x(0)}\left[x(-h]^{2},\right.
$$

$$
(3 / 2-b h) x(0) \leq-\frac{1}{2 x(0)}[x(-h)]^{2}<0
$$


Hence, the described situation can occur only if $b h>3 / 2$, and for $b h \leq 3 / 2$ condition (10) is satisfied, i.e. equation (9) is stable. This statement was proved in [13] in a different way, also on the basis of Razumikhin's method.

Thus, the transition from $N=1$ to $N=2$ widens to one and a half the interval of values $b$ for which the stability of equation (9) is guaranteed with a given $h$. It should be noted that the precise estimate, for which stability takes place, is $b h \leq \pi / 2$, which can be obtained by considering the corresponding characteristic quasipolynomial. Thus Razumikhin's method gives an estimate in this example which differs from the precise one by less than $5 \%$.

It could be shown that the transition to $N=3,4$ etc. leads to further refinements of the estimate. However, this transition would require an essential complication of arguments and apparently noone did it until now.

The point is that the foregoing discussion of the cases $N=1$ and $N=2$ is valid for the RDE

$$
\dot{x}(t)=-b x\left(t-h_{1}(t)\right)
$$

with $0 \leq h_{1}(t) \leq h$ as well. (And it is a strong aspect of Razumikhin's method, because the method of characteristic quasipolynomials is no longer applicable to equation (13).) However, it appears (see [34], §38) that the continuous function $h_{1}(\cdot)$ could be constructed for any $b>3 / 2 h$ for which equation (13) is unstable, i.e. the estimate $b h \leq 3 / 2$, obtained by Razumikhin's method and guaranteeing stability, is best possible for this equation. A refinement of the estimate $b h \leq 3 / 2$ requires arguments which use essentially the constancy of the delay or at least the impossibility of its considerable decrease in comparison with the estimating value.

\section{TRANSFORMATION OF A SYSTEM OF RDES}

We can see from Section 12 that, although an application of Theorem 9 even for $N=1$ leads to more precise estimates than Theorem 7 , it requires essentially more complicated arguments and therefore is poorly algorithmized. For this reason, Razumikhin applied a method of preliminary transformations of the system of RDEs, after which an application of Theorem 7 leads to refined estimates. This transformation, based on Lagrange formula, was applied for other aims also, and now, it is difficult to find out who proposed it first. We shall demonstrate it for RDE (5) from Section 6 .

First of all, note that the sufficient condition of stability obtained in Section 6 for equation (5) by Theorem 7 , can immediately be extended to the equations

$$
\dot{x}(t)=a x(t)+\sum_{i=1}^{k} b_{i} x\left(t-h_{i}\right) \quad\left(k \geq 1, \text { all } h_{i}>0\right)
$$

and has the form $\sum_{i=1}^{k}\left|b_{i}\right| \leq-a$.

Transform now equation (5) using Lagrange formula as follows:

$$
\dot{x}(t)=(a+b) x(t)-b[x(t)-x(t-h)]=(a+b) x(t)-b h \dot{x}(t-\xi)
$$

where $0<\xi<h$. Substitute the value $\dot{x}$ in the right-hand side to get from equation (5),

$$
\dot{x}(t)=(a+b) x(t)-a b h x(t-\xi)-b^{2} h x(t-h-\xi) .
$$

From here we obtain, by virtue of the previous paragraph, the sufficient condition for the stability of equation (5):

$$
a+b+|a b| h+b^{2} h \leq 0 .
$$

Two remarks have to be made with respect to this reasoning. First of all, the value $\xi$ is not 
constant; but we have already mentioned that the conclusion of Theorem 7 remains valid for nonconstant delay also. Further, the function $x(\cdot)$ must have a derivative on $(t-h, t)$ for application of Lagrange theorem, which is guaranteed for $t \geq t_{0}+h$. Thus, we do not apply Theorem 7 but Theorem 9 with $N=1$. However, no individual properties of equation (5) are used here, except that it is an equation of retarded type.

Inequality (14) determines the domain on $a, b$-plane, which is bounded by the line $b$ $=-1 / h$ and the hyperbola $a=b(b h+1) /(b h-1)$; this domain is shaded steeply in Figure 1 . Together with the domain of stability, known from direct application of Theorem 7 (it is shaded slantingly in Figure 1), we obtain an essential extension of the latter domain. The exact domain of stability is shown on Figgure 1 for comparison; it is bounded by dashed lines - straight line $a+b=0$ from above and line $a+b \cos \left(h \sqrt{b^{2}-a^{2}}\right)=0$ from below (see [24], Section 3.2.3).

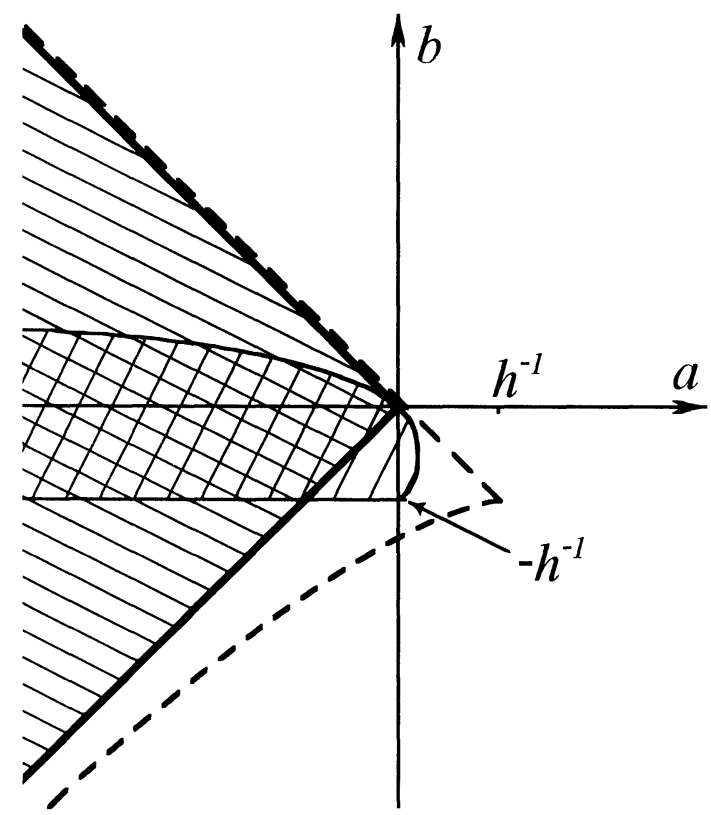

Figure 1

Some applications of Razumikhin's method to determine stability conditions of RDEs can be found in book [38].

\section{OTHER APPLICATIONS OF RAZUMIKHIN'S METHOD}

In this section, we consider some examples of application of Razumikhin's method for other problems.

The following theorem concerns a property similar to exponential stability of the solution $x(t) \equiv 0$ of system $(2)$ with $f(0,0)=0$.

Theorem 10. Assume that the Lyapunov function $V: G \rightarrow \mathbb{R}$ and a number $p>0$ exist such that

$$
\left[V(y)<e^{p h} V(x),(x, y) \in G\right] \Rightarrow[(\operatorname{grad} V(x), f(x, y))+p V(x) \leq 0] .
$$


Then for each $\epsilon>0$ there is a $\delta>0$ such that

$$
\left[|x(t)|<\delta\left(t_{0}-h \leq t \leq t_{0}\right)\right] \Rightarrow\left[V(x(t))<\epsilon e^{-p t} \quad\left(t_{0} \leq t<\infty\right)\right] .
$$

The proof is based on the formulas

$$
\begin{aligned}
& {\left[V(x(t)) e^{p t} \cdot=[(\operatorname{grad} V(x(t)), f(x(t), x(t-h)))+p V(x(t))] e^{p t}\right.} \\
& {\left[V(x(t-h)) e^{p(t-h)}<V(x(t)) e^{p t}\right] \Leftrightarrow\left[V(x(t-h))<V(x(t)) e^{p h}\right]}
\end{aligned}
$$

and on application of Lemma 2 to the function $t \rightarrow V(x(t)) e^{p t}$.

It follows from Theorem 10, for instance, that equation (5) is exponentially stable if $|b|<-a$.

Theorems like Theorem 9 can also be proved about boundedness of solutions and dissipativity of equation (2).

Theorem 11. Let the smooth function $V: \mathbb{R}^{n} \rightarrow \mathbb{R}$ exist such that $V(x) \rightarrow \infty$ for $|x| \rightarrow \infty$ and (grad $V(x), f(x, y)) \leq 0$ for $V(y)<V(x)$ if $|x|$ is sufficiently large. Then $(\forall M>0)(\exists N>0)$ : if $\phi \in K,|\phi(\theta)|<M(-h \leq \theta \leq 0)$, then we have $|x(t)|<N\left(t_{0} \leq t<\infty\right)$ for the solution of problem (2), (3).

Theorem 12. Let all the conditions of Theorem 11 be valid and for any sufficiently large $p>0$, there exists $q>p$, such that $(\operatorname{grad} V(x), f(x, y))<0$, if $V(x)=p, V(y)<q$. Then system (2) is dissipative.

Remark 1. A similar idea can be applied to prove that for any solution $x(\cdot)$ of a system of 1st order RDEs, the point $x(t)$ remains, for $t_{0}<t<\infty$, in a given part of the space if values of the initial function belong to it. Consider e.g. system (2). Let the function $V: \mathbb{R}^{n} \rightarrow \mathbb{R}$ be smooth, $V(x)<0$ in some (connected open) domain $G$ and $V(x)=0$ on its boundary $\partial G$. Let $\phi(\theta) \in \bar{G}:=G \cup \partial G(-h \leq \theta \leq 0)$ and

$$
(x \in \partial G, y \in \bar{G}) \Rightarrow(\operatorname{grad} V(x), f(x, y)) \leq 0 .
$$

Then, from lemma 2, it follows that $x(t) \in \bar{G}$ on the whole interval of existence of the solution $x(\cdot)$ of problem (2),(3). If $\phi(\theta) \in G(-h \leq \theta \leq 0)$ and condition (15) is satisfied, then $x(t) \in G$ on this whole interval.

For example we obtain for the scalar $\operatorname{RDE}(2)$, taking $V(x):=x$, that if $\phi(\theta) \leq 0$ (resp. $<0$ ) for all $\theta \in[-h, 0]$ and $f(0, y) \leq 0$ for all $y \leq 0$, then $x(t) \leq 0$ (resp. $<0$ ) for $t>t_{0}$ on the whole existence interval of the solution $x(\cdot)$ of problem $(2),(3)$.

This remark means in essence that any guiding function for an ODE can be used as a guiding function for an RDE under appropriate assumptions about right-hand sides of both systems. E.g., if applied to systems (1) and (2), the condition ( $\operatorname{grad} V(x), f(x, y)) \leq 0$ must be replaced by

$$
[V(y)<V(x)] \Rightarrow[(\operatorname{grad} V(x), f(x, y)) \leq 0] .
$$

Of course, refinements are possible here in the fashion of Theorem 9.

Remark 2. Razumikhin's method adapted to difference equations (DcEs) turns into a variant of the usual method of mathematical induction. Let us illustrate this on the example of DcE

$$
x_{m+2}=f\left(x_{m}, x_{m+1}\right)(m=0,1, \ldots), \text { where } f: \mathbb{R}^{n} \times \mathbb{R}^{n} \rightarrow \mathbb{R}^{n} .
$$

Let the function $V: \mathbb{R}^{n} \rightarrow \mathbb{R}$ be given. We want to prove that $V\left(x_{m}\right) \leq 0$ for all $m \geq 0$. Then, taking into account discreteness of $m$, the natural analog of Razumikhin's method is the following: we must check that $V\left(x_{m}\right) \leq 0(m=0,1)$, and in addition prove that 


$$
[V(x) \leq 0, V(y) \leq 0] \Rightarrow[V(f(x, y)) \leq 0] \quad\left(\forall x, y \in \mathbb{R}^{n}\right)
$$

But, if we substitute $x=x_{m}, y=x_{m+1}$ here, we obtain the method of induction.

The refinement of estimates in Section 11 can be useful for DcEs as well. Let us consider, for example, the scalar equation

$$
x_{m+2}=a x_{m}+b x_{m+1}(m=0,1, \ldots),
$$

for which the stability domain is

$$
|a| \leq 1,|b| \leq 1-a
$$

The direct approach described in the previous paragraph, with $V(x)=|x|$ or with $V(x)=x^{2}$, gives the smaller domain:

$$
|a|+|b| \leq 1
$$

Let us iterate equation (16) to widen this domain. It gives

$$
x_{m+3}=\left(a+b^{2}\right) x_{m+1}+a b x_{m} .
$$

We obtain from here a condition on $a, b$ which is sufficient for stability of equation (16):

$$
\left|a+b^{2}\right|+|a b| \leq 1
$$

It is not difficult to verify that the $(a, b)$-domain, defined by this inequality, is wider than that of (18), but nevertheless narrower than the exact domain (17).

\section{SUPPLEMENT}

Let us cite some papers $[1-5,7-12,15-20,22-23,29-30,32-33,35-37,39-40,42-48]$, not indicated in [13], where Razumikhin's method is developed and applied, and related problems are considered. (We do not claim that this list of references is complete.)

\section{Acknowledgement}

I am grateful to Professor Mark A. Krasnosel'skii, who suggested to write this paper, and to Professor V.B. Kolmanovskii and Mrs. M.V. Polyakova for their help in its preparation.

\section{REFERENCES}

[1] Bernfeld, S.R. and Haddock, J.R., Lyapunov-Razumikhin functions and convergence of solutions of functional differential equations, Appl. Anal., 9:4 (1979), 235-245.

[2] Bernfeld, S.R., Lakshmikantham, V., and Reddy, Y.M., Fixed point theorems for nonlinear functional operators, Dyn. Syst. Proc. Univ. Fla. Int. Symp., Gainesville, 1976, New York, (1977), 387-389.

[3] Burton, T.A., Perturbations and delays in differential equations, SIAM J. Appl. Math., 29:3 (1975), 422-438.

[4] Chen, Q., A class of Razumikhin-type theorems of stability for super-neutral functional 
differential equations, J. Hunan Univ., 13:3 (1986), 190-199 (in Chinese).

[5] Cheng, M.-H., On Razumikhin-type stability conditions for stochastic functional differential equations, Math. Modell., 5:5 (1984), 299-307.

[6] El'sgol'ts, L.E., Stability of solutions of differential-difference equations, Uspekhi Mat. Nauk. (Sov. Math. Surveys), 9:4 (1954), 95-112 (in Russian).

[7] Fonda, A., Guiding functions and periodic solutions of functional differential equations, Proc. Amer. Math. Soc., 99:1 (1987), 79-85.

[8] Furumochi, T., Periodic solutions of functional differential equations, Lect. Notes Math., 1017 (1983), 194-200.

[9] Furumochi, T., Periodic solutions of functional differential equations with large delays, Funkc. Ekvacioj, 25:1 (1982), 33-42.

[10] Gromova, P.S., Method of Lyapunov vector functions for systems with deviating argument, Direct Method in Stability Theory and its Appl., Novosibirsk, (1981), 46-54 (in Russian).

[11] Gromova, P.S. and Lisina, P.M., Method of Lyapunov vector functions for systems with delay, Different. Equat. with Deviating Arg., 11 (1979), 14-22 (in Russian).

[12] Haddock, J.R., and Terjéki, J., Lyapunov-Razumikhin functions and an invariance principle for functioanl differential equations, J. Differ. Equat., 48:1 (1983) 95-122.

[13] Hale, J., Theory of Functional Differential Equations, 2nd ed., Springer-Verlag, New York 1977.

[14] Hale, J., and Lunel, S.M.V., Introduction to Functional Differential Equations, SpringerVerlag, New York 1993.

[15] Hu, M., Lyapunov functions for neutral functional differential equations, Ann. Differ. Equat., 2:2 (1986), 129-140.

[16] Husainov, L.Ya., On investigation of stability of solutions of differential-functional equations by the method of Lyapunov functions, 9 Intern. Conf. on Nonlin. Osc. Kiev, 30 Aug. - 6 Sept., 1981, V. 2, Kiev (1984), 413-415 (in Russian).

[17] Karatueva, N.A., Method of Lyapunov vector functions for systems of differential equations with delay, Meth. of Lyapunov Functions and its Appl., Novosibirsk (1984), 4958 (in Russian).

[18] Kato, J., Lyapunov's second method in functional differential equations, Tohoku. Math. J, 32:4 (1980), 487-497.

[19] Kato, J., Stability problem in functional differential equations with infinit delay, Funkc. Ekvacioj, 21:1 (1978), 63-80.

[20] Kato, J., Razumikhin type theorem for differential equations with infinit delay, Dyn. Syst. Proc. Univ. Fla. Int. Symp., Gainesville, 1976, 441-443, New York 1977.

[21] Kato, J., On Lyapunov-Razumikhin type theorems for functional differential equations, Funkc. Ekvacioj, 16 (1973), 225-239.

[22] Kato, J., On Lyapunov-Razumikhin type theorems, Lect. Notes Math., 243 (1971), 54-65.

[23] Kim, A.V., To Lyapunov functions method for systems with aftereffect, Meth. of Lyapunov Functions in Anal. of Dynam. Systems, Novosibirsk (1987), 79-83, (in Russian).

[24] Kolmanovskii, V.B. and Myshkis, A.D., Applied Theory of Functional Differential Equations, Kluwer, Dordrecht 1992 .

[25] Kolmanovskii, V.B. and Nosov, V.R., Stability of Functional Differential Equations, Academic Press, New York, London 1986.

[26] Kolmanovskii, V.B. and Nosov, V.R., Stability and Periodic Solutions of Regulated Systems with Delay, Nauka, Moscow 1981 (in Russian).

[27] Krasovskii, N.N., Stability of Motion, Stanford University Press 1963.

[28] Krasovskii, N.N., On application of Lyapunov's second method to equations with time delays, Prikl. Mat. i Mekh. (Appl. Math \& Mech), 20:2 (1956), 315-327.

[29] Kricztin, T., On the rate of convergence of solutions of functional differential equations, Funkc. Ekvacioj, 29:1 (1986), 1-10. 
[30] Li, S., A V-function method for stability of functional differential equations and its applications, Sci. Sin., A30:5 (1987), 449-461.

[31] Lyapunov, A.M., Collected Works, B.2, Nauka, Moscow 1956 (in Russian).

[32] Mikolajska, Z., Remarque sur la stabilité d'une solution du systéme d'équations différentielles à paramètre retardé, Colloq. Math, 18 (1967), 59-66.

[33] Misnik, A.F. and Nosov, V.R., To the Question on Stability of Differential-Difference Equations of Neutral Type, Collect. of Sc. Works of post-grad. students, P. Lumumba Univ. of Friendship of People, Dept. of Phys.-Math. and Natuar. Sci., 1 (1968), 43-55 (in Russian).

[34] Myshkis, A.D., Linear Differential Equations with Retarded Arguments, 2nd ed., Nauka, Moscow 1972 (in Russian).

[35] Prasolov, A.V., On application of special form Lyapunov functions for systems with aftereffect, Math. Theory of Control of Techn. Objects, Leningrad (1982), 5-12 (in Russian).

[36] Prasolov, A.V., On application of Lyapunov functions for investigation of instability of solutions of systems with aftereffect, Vestn. LGU (Bull. Leningrad State Univ.), No.19 (1981), 116-118 (in Russian).

[37] Rao, D.R., Another method of studying the stability of the solutions of neutral functional differential systems, Proc. Nat. Inst. Sci. India, A34:5 (1968), 238-241.

[38] Razumikhin, B.S., Stability of Hereditary Systems, Nauka, Moscow 1988 (in Russian).

[39] Razumikhin, B.S., Direct method of investigation of stability of systems with aftereffect, Preprint, Moscow, Res. Inst Syst. Analysis (1984), 1-75.

[40] Razumikhin, B.S., A method of investigation of stability of systems with aftereffect, Doklady AN SSSR, 167:6 (1966), 1234-1237 (in Russian).

[41] Razumikhin, B.S., On stability of systems with delay, Prykl. Mat. i Mekh. (Appl. Math E Mech.) 20, N4, 500-512, (1956), (in Russian).

[42] Seborg, D.E. and Johnson, E.F., Construction of regions of stability for non-linear systems containing time delays, Int. J. Contr, 12:6 (1970), 961-976.

[43] Sèifert, G., Lyapunov-Razumikhin conditions for asymptotic stability in functional differential equations of Volterra type, J. Different. Equat. 16:2 (1974), 289-297.

[44] Seifert, G., Lyapunov-Razumikhin conditions for stability and boundedness of functional differential equations of Volterra type, J. Different Equat., 14:3 (1973), 424-430.

[45] Terjéki, J., On the asymptotic stability of solutions of functional differential equations, Ann. Pol. Math., 36:3 (1979), 299-314.

[46] Winston, E., Asymptotic stability for ordinary dufferential equations with delayed perturbations, SIAM J. Math. Anal., 5:2 (1974), 303-308.

[47] Xia, X., Razumikhin type theorems by Lyapunov functional in neutral functional differential equations, Chin. Ann. Math A, 9:4 (1988), 371-379 (in Chinese).

[48] Zhang, B., Razumikhin-type theorem in functional differential equations with infinite delay, Ann. Differ. Equat., 2:1 (1986), 87-101. 


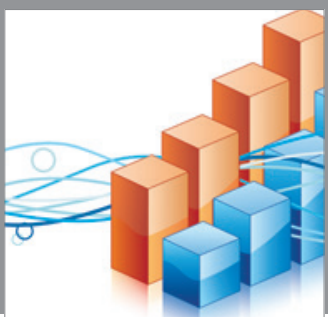

Advances in

Operations Research

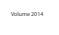

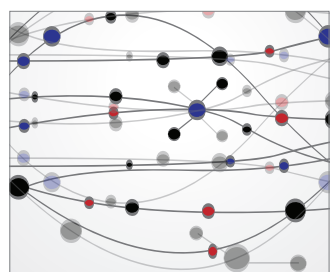

\section{The Scientific} World Journal
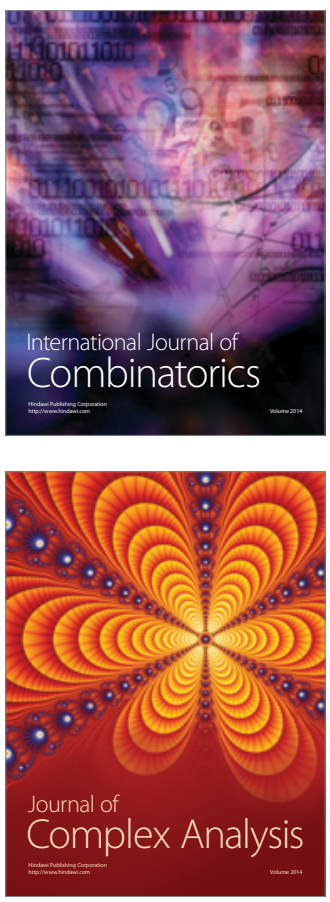

International Journal of

Mathematics and

Mathematical

Sciences
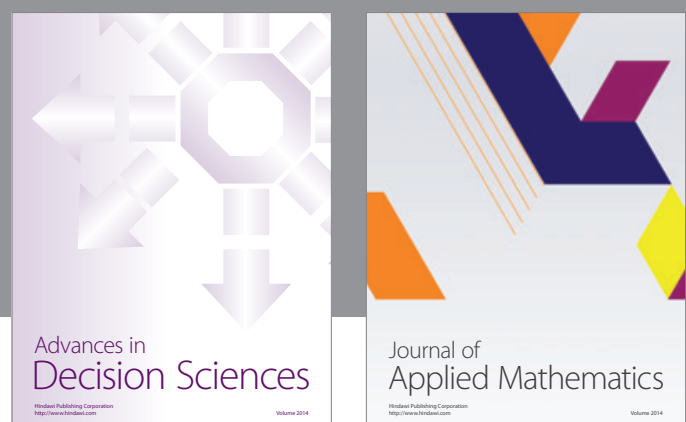

Journal of

Applied Mathematics
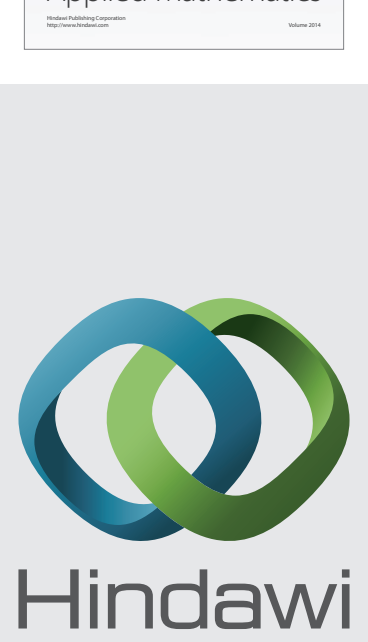

Submit your manuscripts at http://www.hindawi.com
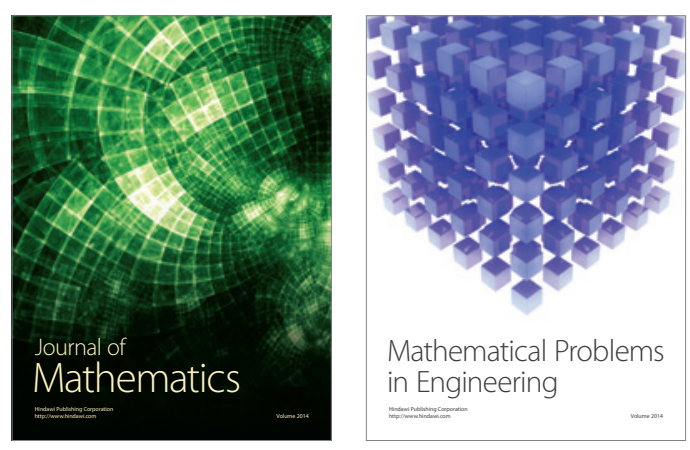

Mathematical Problems in Engineering
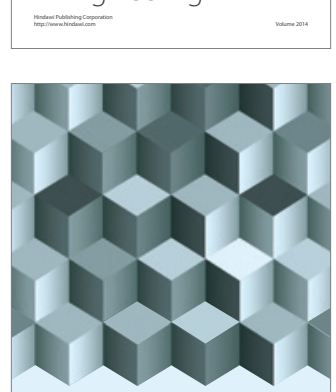

Journal of

Function Spaces
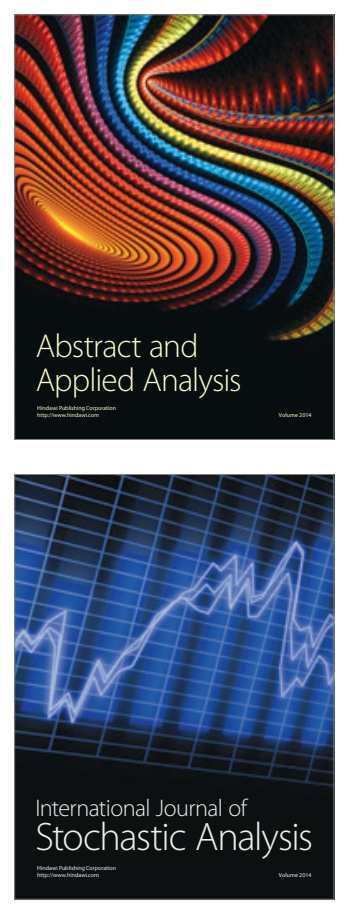

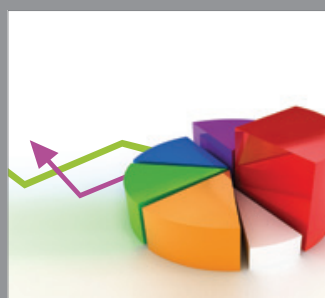

ournal of

Probability and Statistics

Promensencen
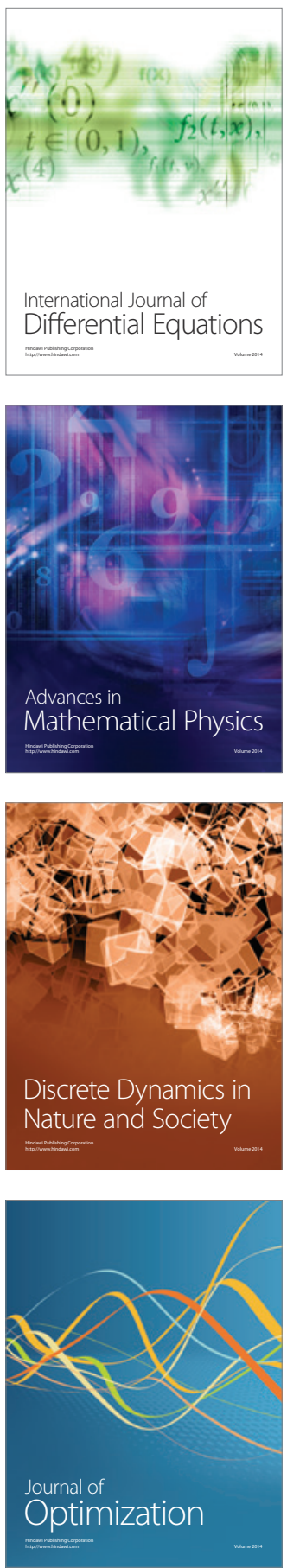